\title{
Chemoradiotherapy alone vs. chemoradiotherapy and hysterectomy for locally advanced cervical cancer: A systematic review and updated meta-analysis
}

\author{
WEIJIA LU ${ }^{1}$, CANXIONG LU ${ }^{2}$, ZHIWU YU ${ }^{3}$ and LEI GAO ${ }^{4}$ \\ ${ }^{1}$ The Second Clinical Medical College, Guangzhou University of Chinese Medicine, Guangzhou, Guangdong 510006; \\ ${ }^{2}$ Department of Laboratory, Foshan Sanshui Hospital of Chinese Medicine, Foshan, Guangdong 528100; \\ ${ }^{3}$ Division of Laboratory Science, Affiliated Cancer Hospital \& Institute of Guangzhou Medical University, \\ Guangzhou, Guangdong 510095; ${ }^{4}$ Department of Radiotherapy, The Second Affiliated Hospital of \\ Guangzhou University of Chinese Medicine, Guangzhou, Guangdong 510006, P.R. China
}

Received May 4, 2020; Accepted November 18, 2020

DOI: $10.3892 / \mathrm{ol} .2020 .12421$

\begin{abstract}
The benefit of adjuvant hysterectomy after definitive concurrent chemoradiotherapy (CCRT) for locally-advanced cervical cancer (LACC) is controversial. The purpose of the present study was to systematically search the literature and perform a meta-analysis to compare overall survival (OS) and disease-free survival (DFS) between patients subjected to CCRT with hysterectomy and those who underwent CCRT alone. The PubMed, Scopus, Embase and Google scholar databases were searched. A meta-analysis to determine hazard ratios (HRs) and odds ratios (ORs) with meta-regression was performed for the following moderators: Disease stage, histology and proportion of radical hysterectomy. Data from 14 studies were included. The results indicated that patients who received CCRT with hysterectomy had significantly better OS (HR, 0.72; 95\% CI, 0.56 to $0.91 ; \mathrm{I}^{2}=19 \% ; \mathrm{P}=0.007$ ) and DFS (HR, $0.72 ; 95 \% \mathrm{CI}, 0.56$ to $0.93 ; \mathrm{I}^{2}=27 \% ; \mathrm{P}=0.01$ ) than those treated with CCRT alone. However, in a subgroup analysis by study type, the results were significant only for retrospective studies but not for randomized controlled trials (RCTs). However, only 2 RCTs were included with small sample size, heterogeneity and low overall quality. Subgroup analyses based on the use of brachytherapy in the CCRT with hysterectomy group demonstrated no difference in OS and DFS between the two groups. Regarding the absolute numbers of death and recurrence events, no significant difference in mortality (OR, $0.91 ; 95 \% \mathrm{CI}, 0.62$ to $1.33 ; \mathrm{I}^{2}=0 \% ; \mathrm{P}=0.64$ ) was determined between the two groups, but a significantly
\end{abstract}

Correspondence to: Dr Lei Gao, Department of Radiotherapy, The Second Affiliated Hospital of Guangzhou University of Chinese Medicine, 55 Neihuanxi Road, Guangzhou, Guangdong 510006, P.R. China

E-mail: 707565065@qq.com

Key words: cervical cancer, radiotherapy, surgery, chemoradiotherapy, survival, recurrence reduced incidence of recurrence was observed in the CCRT with hysterectomy group (OR, 0.61; 95\% CI, 0.47-0.79; $\mathrm{I}^{2}=29 \%$; $\mathrm{P}=0.0002$ ). The meta-regression results point to a significant influence of the proportion of stage II patients on OS. Despite the overall analysis indicating improved OS and DFS with the use of adjuvant hysterectomy after CCRT, subgroup analysis based on similar treatment protocols failed to demonstrate any significant benefit of hysterectomy in LACC. However, the results indicated that the recurrence rate may be higher in patients undergoing CCRT without hysterectomy. The limited quality of the studies included and selection bias from retrospective studies restrict the possibility to draw strong conclusions.

\section{Introduction}

Data from the recent GLOBOCAN report suggests that cervical cancer is the fourth most common malignancy in females worldwide and the second most common cancer type in low- and middle-income countries (1). Globally, a total of 569,847 new cases of cervical cancer were reported in 2018 and an estimated 311,365 deaths (1). According to a recent systematic review on cervical cancer, it is one of the leading causes of death amongst females in developing countries (2).

According to the International Federation of Gynecology and Obstetrics classification, stage IB2, IIA2, IIB, IIIA, IIIB and IVA cervical cancer is defined as locally advanced cervical cancer (LACC) (3). The treatment of choice for patients with such a disease is definitive concurrent chemoradiotherapy (CCRT), consisting of external beam radiotherapy with concomitant platinum-based chemotherapy and with or without further interstitial brachytherapy $(4,5)$. Despite the better survival it achieves compared to radiotherapy alone, the 5-year survival for patients remains $\sim 70 \%$ (6). Completion or so-called 'adjuvant' hysterectomy has been used after CCRT in certain patients to improve survival (7). However, no consensus over the benefit of such adjuvant surgery in LACC has been reached in the literature (8). Advocates of additional surgery argue that early hysterectomy leads to better disease control and avoidance of more extensive surgery, such as pelvic 
exenteration, in the event of a future recurrence $(7,9)$. On the other hand, adjuvant hysterectomy is not morbidity-free and the rate of grade-3 adverse events may be as high as $26.5 \%$ (8).

So far, two meta-analyses have performed data syntheses in an attempt to provide evidence on the benefit of hysterectomy with CCRT for LACC. Shim et al (8) performed a meta-analysis of studies published until April 2016 and compiled survival and recurrence data from eight studies comparing CCRT with hysterectomy vs. CCRT alone. However, they only pooled the odds ratio (OR) data in their analysis. Shi et al (10) provided another meta-analysis of studies published until August 2017, pooling eight studies that used both radiotherapy alone and CCRT with or without hysterectomy. Their study only analyzed survival data to obtain hazard ratios (HRs) without comparing recurrence rates. The publication of new studies since then $(11,12)$ warrants a more comprehensive and updated analysis to guide clinicians treating LACC. Therefore, the purpose of the present study was to systematically search the literature and conduct a comprehensive meta-analysis with meta-regression to compare survival and recurrence between patients undergoing CCRT with hysterectomy and those undergoing CCRT alone.

\section{Materials and methods}

Inclusion criteria. The Preferred Reporting Items for Systematic Reviews and Meta-analyses (PRISMA) (13) guidelines were followed during the preparation of this review. The protocol of the study was, however, not registered. All types of peer-reviewed studies conducted on patients with LACC i.e., stages IB2 to IVA, were included. The included studies compared groups of patients undergoing CCRT with hysterectomy with those undergoing CCRT alone and reported on overall survival (OS) and/or disease-free survival (DFS) as outcome variables. Both groups of patients included patients who underwent brachytherapy, who had radical/simple hysterectomy and/or who had different histological types of LACC. Studies including other cancer stages such as IB1 or IVB were only considered if the overall percentage of patients with these stages was $<10 \%$ of the study sample. The criteria for exclusion were as follows: i) Non-comparative single-arm studies; ii) studies using only chemotherapy or radiotherapy and not combined CCRT; iii) case series, case reports, letters to editors and abstracts; iv) non-English language studies and articles with unavailable full-text versions. In cases of studies with overlapping data, only the study reporting the largest dataset was included.

Search strategy. An electronic search of the PubMed, Scopus, Embase and Google scholar databases was performed. The last search was performed on the 20th of February, 2020. A total of two independent reviewers (CL, ZY) performed the database search using the following keywords in various combinations: 'Cervical cancer', 'locally advanced cervical cancer', 'chemoradiotherapy', 'chemotherapy', 'radiotherapy', 'surgery', 'hysterectomy' and 'brachytherapy'. The exact search terms and the number of results obtained are presented in Table SI. After the selection of studies, the bibliography of all included studies was manually checked to identify any studies missed during the digital search. First, two different reviewers
(CL, ZY) screened the titles and abstracts of search records. After screening of abstracts, the full texts of relevant articles were extracted and assessed in detail based on the inclusion criteria. Any disagreements during the study selection process were resolved by discussion with the third reviewer (LG).

Data extraction and quality of included studies. A data abstraction form was used to extract the following details: Author name, publication year, study type, sample size, demographic data, LACC stage, histological type, lymph node metastasis, CCRT protocol, brachytherapy details, chemotherapy regimen, hysterectomy type and timing, follow-up and study outcomes. The primary outcomes of interest were the differences in OS and DFS between patients with LACC undergoing CCRT with hysterectomy and those undergoing CCRT alone using HRs. The secondary outcomes of interest were differences in mortality and recurrence (both local and distant) between the two groups by comparing ORs.

The Cochrane Collaboration Risk Assessment Tool was used to assess the risk of bias in randomized controlled trials (RCTs) (14). For non-RCTs, the risk of a bias assessment tool for non-randomized studies was used (15). For each domain, studies were graded as having low, high or unclear risk of bias.

Statistical analysis. The software Review Manager [version 5.3; Nordic Cochrane Centre (Cochrane Collaboration)] was used for the meta-analysis. HR values for OS and DFS, as well as data on the absolute number of deaths and recurrences, were extracted from the studies included. In cases in which HR values were unavailable, they were extrapolated from Kaplan-Meier survival curves using the Microsoft Excel sheet developed by Matthew Sydes and Jayne Tierney (16). The study estimates were then combined using inverse variance-weighted averages of logarithmic HRs in a random-effects model. Categorical data of mortality and any recurrences were summarized using the Mantel-Haenszel OR with $95 \%$ confidence intervals (CI) in a random-effects model. Heterogeneity was calculated using the $\mathrm{I}^{2}$ statistic. Based on $\mathrm{I}^{2}$ values, heterogeneity was classified as low (25-50\%) medium (50-75\%) or substantial ( $>75 \%)$. Subgroup analyses by study type and use of brachytherapy in the CCRT with hysterectomy group were performed using a random effects model. A sensitivity analysis was conducted to assess the influence of each study on the overall estimate by excluding one study at a time. Publication bias was assessed based on visual inspection of funnel plots only if $>10$ studies had been included in the analysis. In addition, a meta-regression analysis was performed using a random-effects model with Open MetaAnalyst software (17). The influence on the pooled effect size of the proportion of patients with stage IB2, stage II, stage III and squamous cell carcinoma (SCC), as well as the proportion of patients undergoing radical hysterectomy, were analyzed using a univariate meta-regression analysis. Meta-regression coefficients (Beta) with 95\% CIs were calculated. Since $\log H R / \log O R>0$ corresponds to HR/OR $>1$ and $\log \mathrm{HR} / \mathrm{OR}<0$ corresponds to $\mathrm{HR} / \mathrm{OR}<1$, a negative meta-regression coefficient indicates that HR/OR decreases as a given factor increases, and a positive coefficient indicates that HR/OR increases as a given factor increases. 


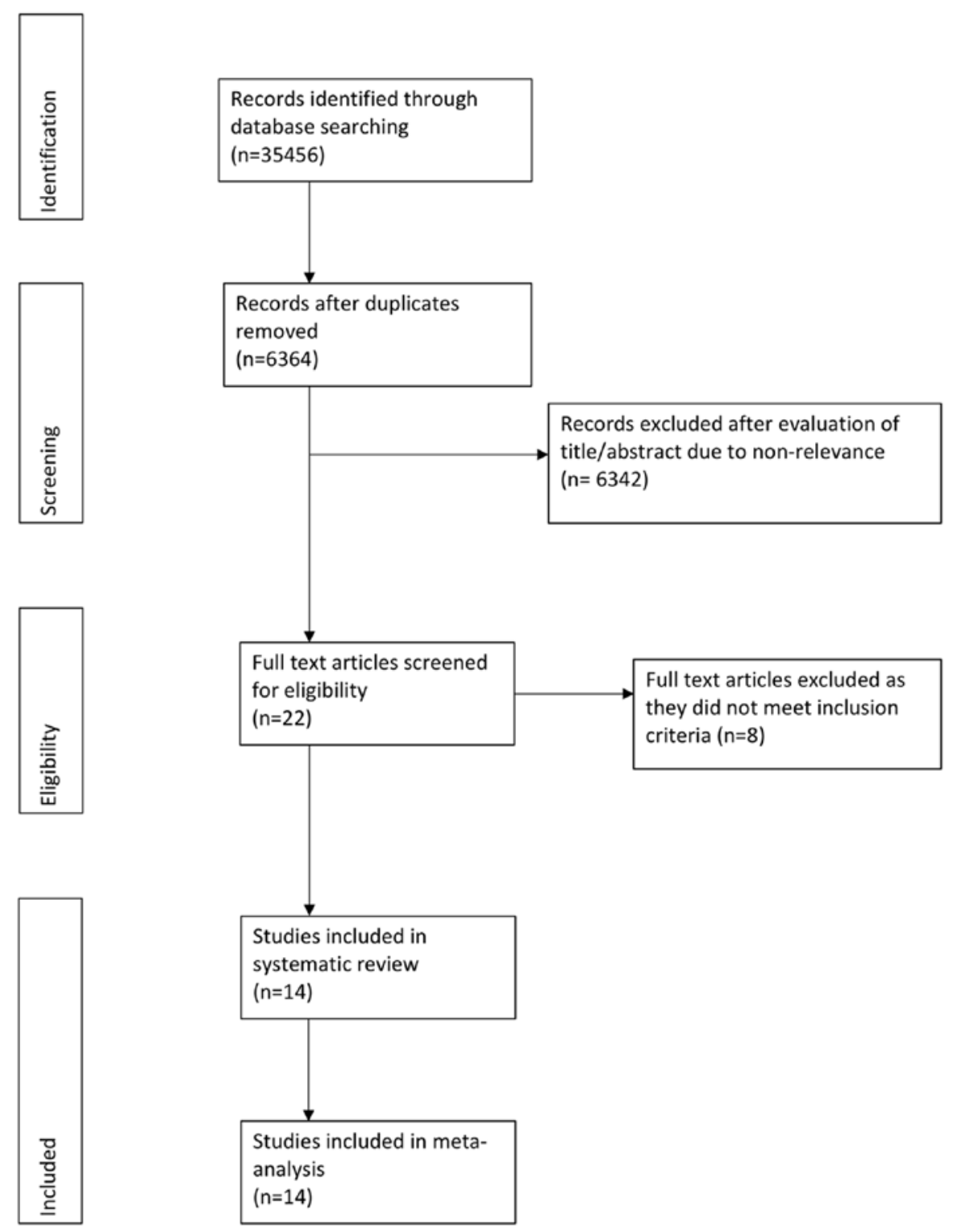

Figure 1. Study flow-chart.

\section{Results}

Study selection. Fig. 1 presents the PRISMA flow chart of the search and selection process. Of the eligible records identified, 22 were subjected to full-text review, eight of which $(7,9,18-23)$ were excluded for the following reasons: A total of two studies used only radiotherapy $(22,23)$, two were non-comparative studies $(7,19)$, one study included a large cohort of patients with stage I cancer (21), one study evaluated patients with stage IVB cervical cancer (18), one study had duplicate data (9) and the full-text of one study was unavailable (20). Finally, data from 14 studies were included in this systematic review and meta-analysis $(11,12,24-35)$.

Table I presents details of the included studies. Except for 2 RCTs $(31,32)$, all were retrospective cohort studies. From these 14 studies, the data of 1,224 patients undergoing CCRT and hysterectomy were compared with those of 2,635 patients undergoing CCRT alone. The sample size of the CCRT with hysterectomy groups ranged from 24 to 192 patients, while the sample size of the CCRT groups ranged from 30 to 1,407 patients. The median age of the patients was $\geq 45$ years in all studies. None of the studies reported any statistically significant differences in the distribution of cancer stages between the two groups. None of the studies reported any statistically significant differences in tumor histology between the two groups, except for one study (12). In addition, one study reported a significant difference in lymph node metastasis between the two groups at baseline (28). Furthermore, four studies $(25,27,29,31)$ did not use brachytherapy in the CCRT with hysterectomy group and used the procedure only in the CCRT group. The chemotherapy regimens included mostly cisplatin $\left(40 \mathrm{mg} / \mathrm{m}^{2}\right.$ weekly) with certain variations across studies. Of note, two studies $(29,33)$ carried out hysterectomies only in good responders, defined as patients with a decrease in tumor volume of at least $50 \%$ according to MRI on 


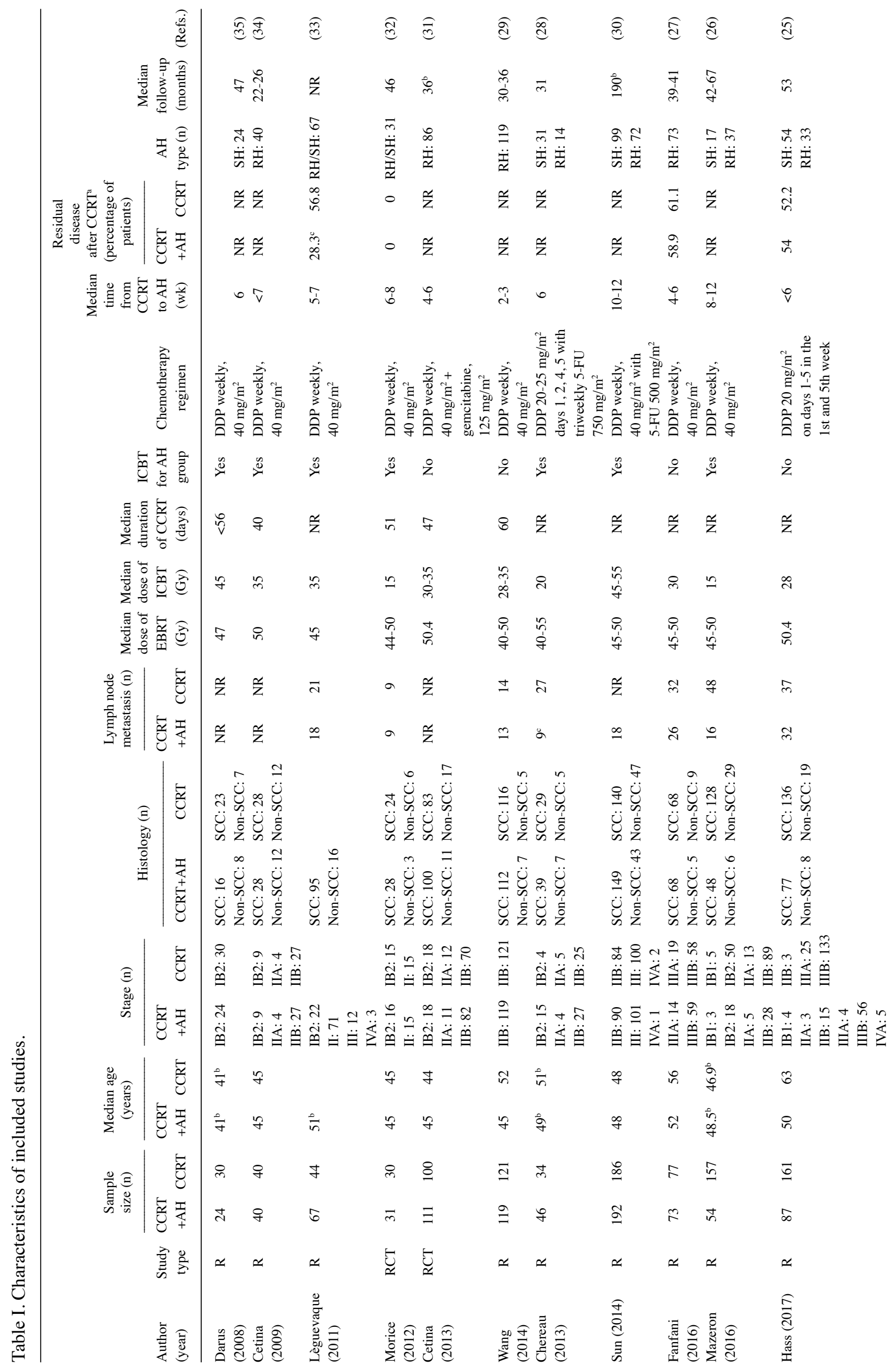




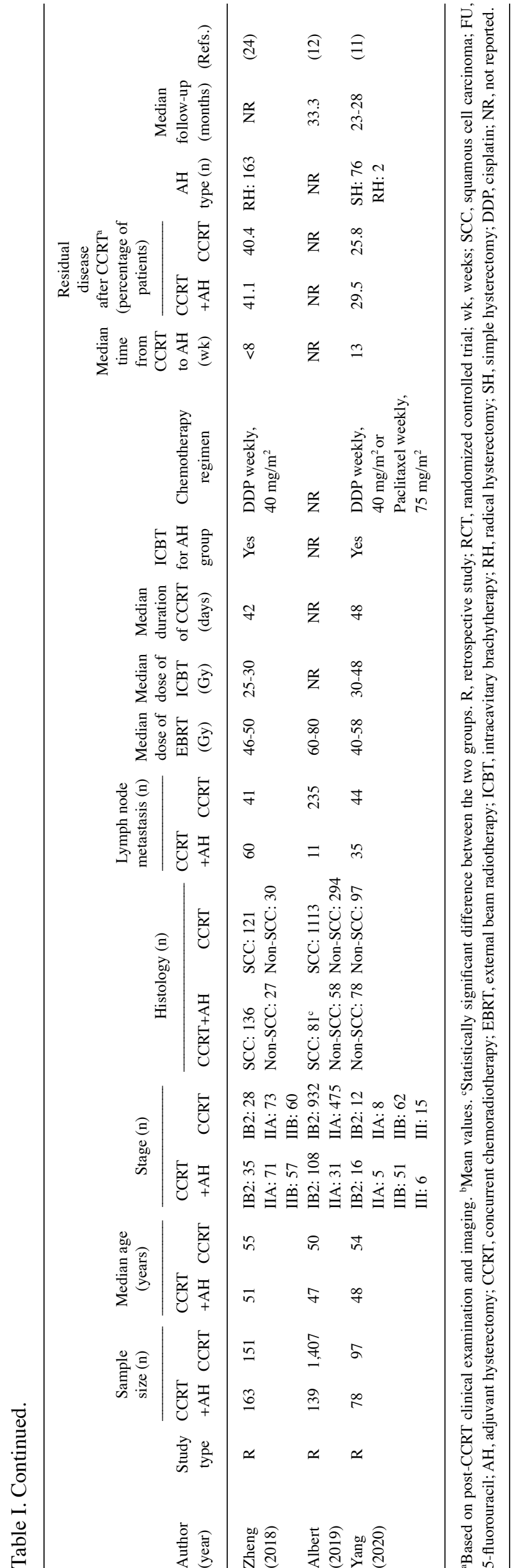

CCRT completion, while one RCT (32) conducted hysterectomies only in complete responders (patients without residual disease). Most studies did not report data on the percentage of patients with residual disease after CCRT. Both simple and radical hysterectomies were used across studies at varying proportions.

Meta-analysis. After pooling data from 12 studies, it was determined that patients receiving CCRT with hysterectomy had significantly better OS than those undergoing CCRT alone (HR, 0.72; 95\% CI, 0.56 to $0.91 ; \mathrm{I}^{2}=19 \% ; \mathrm{P}=0.007$; Fig. 2). The funnel plot did not indicate any gross asymmetries (Fig. S1). However, on subgroup analysis, the difference was significant only for retrospective studies (HR, 0.69; 95\% CI, $0.55-0.87 ; \mathrm{I}^{2}=9 \% ; \mathrm{P}=0.002$ ) but not for the two RCTs (HR, 1.36; 95\% CI, 0.27-6.88; $\mathrm{I}^{2}=67 \%$; $\mathrm{P}=0.71$; Fig. 2). Similarly, after dividing studies based on the use of brachytherapy in the CCRT and hysterectomy group, no statistically significant difference in OS was obtained in any of the subgroups (without brachytherapy: HR, $0.89 ; 95 \% \mathrm{CI}, 0.57-1.39 ; \mathrm{I}^{2}=0 \% ; \mathrm{P}=0.61$; with brachytherapy: HR, 0.70; 95\% CI, 0.49-1.01; $\mathrm{I}^{2}=33 \%$; $\mathrm{P}=0.06$; Fig. 3).

A similar pattern was obtained after pooling data from 10 studies in a meta-analysis for DFS. An overall significant effect in favor of CCRT with hysterectomy was determined, suggesting improved DFS with hysterectomy (HR, 0.72; 95\% CI, 0.56 to $0.93 ; \mathrm{I}^{2}=27 \%$; $\mathrm{P}=0.01$; Fig. 4). However, on subgroup analysis, the significant effect was restricted to retrospective studies only (HR, $0.68 ; 95 \% \mathrm{CI}, 0.53-0.88$, $\mathrm{I}^{2}=12 \%$; $\mathrm{P}=0.003$ ) with no significant differences in DFS in RCTs (HR, 1.10; 95\% CI, 0.33-3.63; $\mathrm{I}^{2}=72 \%$; $\mathrm{P}=0.88$; Fig. 4). No evidence of publication bias was observed in the funnel plot (Fig. S2). After further dividing the studies based on the use of post-CCRT brachytherapy in the CCRT with hysterectomy group, no differences in DFS were obtained between the two groups with the use of brachytherapy (HR, 0.78; 95\% CI, 0.55-1.11; $\left.\mathrm{I}^{2}=39 \% ; \mathrm{P}=0.17\right)$ or without the use of brachytherapy (HR, $0.68 ; 95 \%$ CI, $0.41-1.13 ; \mathrm{I}^{2}=43 \%$; $\mathrm{P}=0.13$; Fig. 5).

A total of seven studies reported data on the number of deaths in their study population. The mortality was $19.4 \%$ in the CCRT with hysterectomy group and $20.6 \%$ in the CCRT only group without any statistically significant differences (OR, 0.91; 95\% CI, 0.62-1.33; $\mathrm{I}^{2}=0 \%$; $\mathrm{P}=0.64$; Fig. 6). Data on recurrence were provided by 13 studies. Pooled analysis indicated a statistically significant reduction in the incidence of recurrence in the CCRT with hysterectomy group (17.14 vs. 26.96\%; OR, 0.61; 95\% CI, 0.47 to 0.79; $\mathrm{I}^{2}=29 \%$; $\mathrm{P}=0.0002$; Fig. 7). Again, this result was significant only for retrospective studies (OR, 0.56; 95\% CI, 0.44-0.72; $\mathrm{I}^{2}=19 \%$; $\mathrm{P}<0.0001$ ) but not for RCTs (OR, 1.14; 95\% CI, 0.40-3.26; $\mathrm{I}^{2}=49 \%$; $\mathrm{P}=0.80$; Fig. 7). The funnel plot did not indicate any gross asymmetries (Fig. S3). On further subgroup analysis based on the addition of brachytherapy in the CCRT with hysterectomy group, recurrence rates were significantly lower in the CCRT with hysterectomy group with brachytherapy (OR, 0.63 ; 95\% CI, 0.42-0.94; $\mathrm{I}^{2}=50 \% ; \mathrm{P}=0.03$ ) and in the CCRT with hysterectomy group without brachytherapy (OR, 0.62 ; 95\% CI, 0.44-0.88; $\left.\mathrm{I}^{2}=0 \% ; \mathrm{P}=0.007\right)$ than in the CCRT alone group (Fig. 8). 


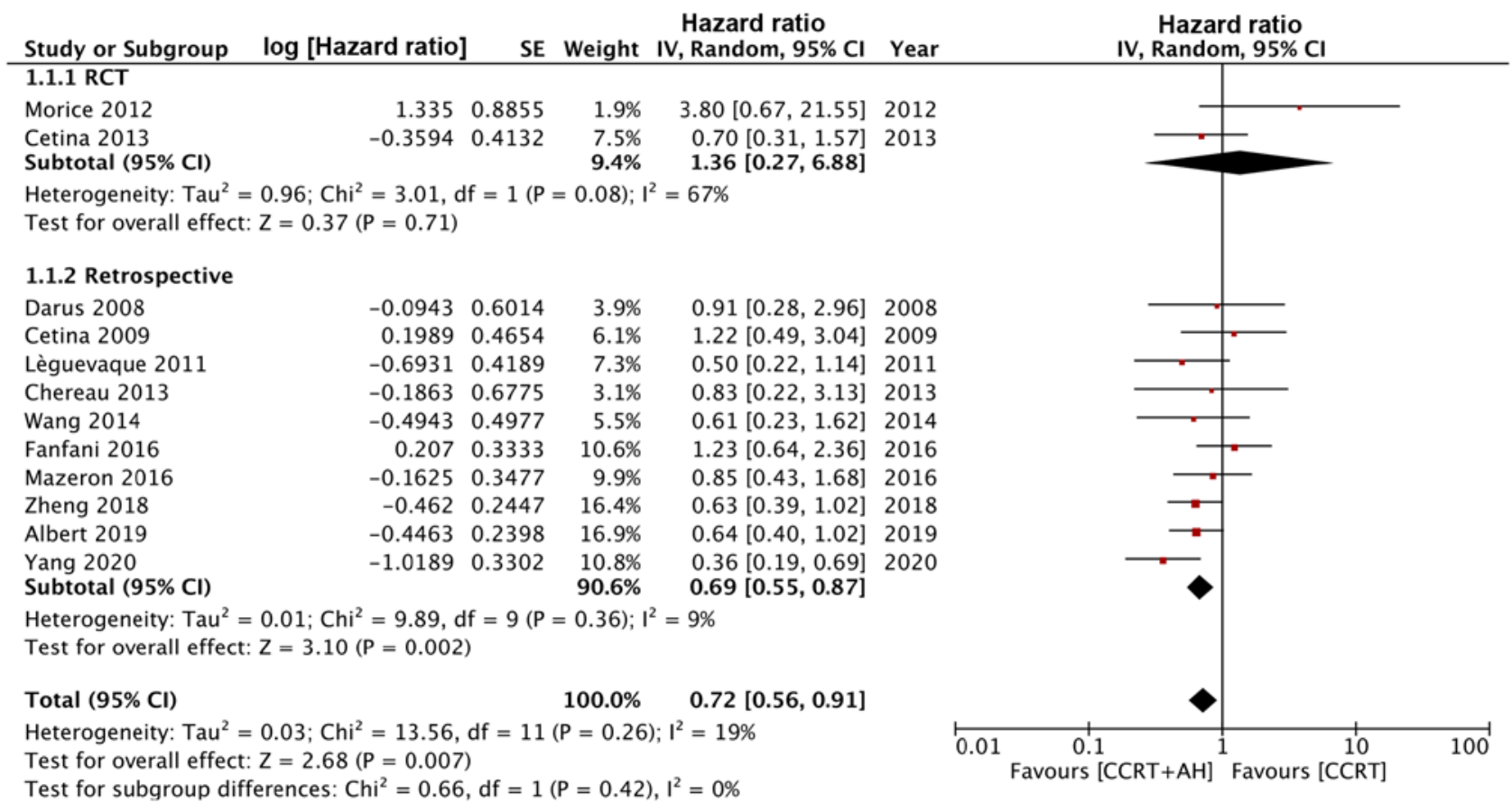

Figure 2. Forest plot of overall survival for CCRT with AH vs. CCRT alone with subgroup analysis based on study type. CCRT, concurrent chemoradiotherapy; $\mathrm{AH}$, adjuvant hysterectomy; RCT, randomized controlled trial; SE, standard error; IV, inverse variance; df, degrees of freedom.

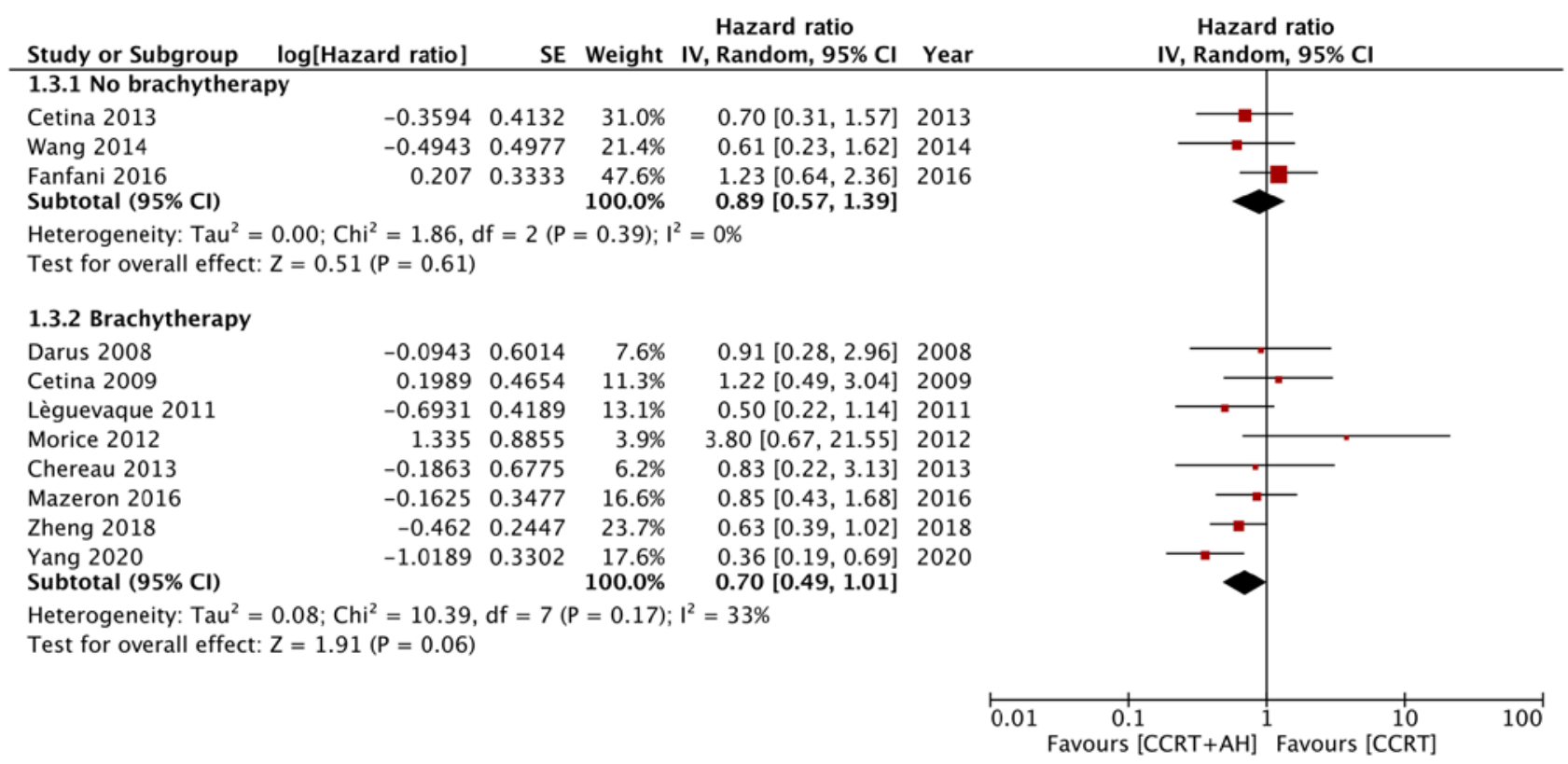

Figure 3. Forest plot of overall survival for CCRT with AH vs. CCRT alone with subgroup analysis based on the use of brachytherapy in the CCRT with hysterectomy group. CCRT, concurrent chemoradiotherapy; AH, adjuvant hysterectomy; SE, standard error; IV, inverse variance; df, degrees of freedom.

Sensitivity and meta-regression analysis. Both primary and secondary outcomes remained the same after each one-at-a-time exclusion of singular studies. The influence of the proportion of patients with stage IB2, stage II, stage III, SCC and radical hysterectomy on the pooled effect size was analyzed. The results are presented in Table II. The OS was significantly influenced by the proportion of stage II patients. The log HR decreased as the proportion of stage II patients increased, indicating improved OS with CCRT and hysterectomy (Fig. 9).
Risk of bias analysis. Table SII presents the results of the assessments of the risk of bias in the RCTs and retrospective studies. The RCTs included failed to clearly describe randomization, allocation concealment and blinding of outcome assessment. The trial of Cetina et al (31) had high number of patients lost to follow up Among the retrospective studies, the selection of participants was from multiple hospital settings in four studies (12,25-27); only one study (34) performed baseline matching of study cohorts, while two studies $(11,12)$ presented multivariate-adjusted HRs. 


\begin{tabular}{|c|c|c|c|c|c|c|c|}
\hline Study or Subgroup & log [Hazard ratio] & SE & Weight & $\begin{array}{l}\text { Hazard ratio } \\
\text { IV, Random, } 95 \% \mathrm{Cl}\end{array}$ & $\begin{array}{r}\text { Hazard } \\
\text { IV, Rando }\end{array}$ & ratio & \\
\hline \multicolumn{8}{|l|}{ 1.2.1 RCT } \\
\hline Cetina 2013 & -0.4283 & 0.3165 & $11.8 \%$ & $0.65[0.35,1.21]$ & & & \\
\hline $\begin{array}{l}\text { Morice } 2012 \\
\text { Subtotal }(95 \% \mathrm{Cl})\end{array}$ & 0.802 & 0.5698 & $\begin{array}{r}4.6 \% \\
16.4 \%\end{array}$ & $\begin{array}{l}2.23[0.73,6.81] \\
1.10[0.33,3.63]\end{array}$ & & & \\
\hline \multicolumn{8}{|c|}{$\begin{array}{l}\text { Heterogeneity: } \text { Tau }^{2}=0.54 ; \mathrm{Chi}^{2}=3.56, \mathrm{df}=1(\mathrm{P}=0.06) ; \mathrm{I}^{2}=72 \% \\
\text { Test for overall effect: } \mathrm{Z}=0.16(\mathrm{P}=0.88)\end{array}$} \\
\hline \multicolumn{8}{|l|}{ 1.2.2 Retrospective } \\
\hline Cetina 2009 & 0.239 & 0.5294 & $5.2 \%$ & $1.27[0.45,3.58]$ & & & \\
\hline Darus 2008 & -0.5798 & 0.7451 & $2.8 \%$ & $0.56[0.13,2.41]$ & & & \\
\hline Fanfani 2016 & -0.1744 & 0.275 & $14.2 \%$ & $0.84[0.49,1.44]$ & & & \\
\hline Lèguevaque 2011 & -0.3857 & 0.2837 & $13.6 \%$ & $0.68[0.39,1.19]$ & & & \\
\hline Mazeron 2016 & 0.0296 & 0.3109 & $12.1 \%$ & $1.03[0.56,1.89]$ & & & \\
\hline Wang 2014 & -0.9163 & 0.4074 & $8.1 \%$ & $0.40[0.18,0.89]$ & & & \\
\hline Yang 2020 & -0.7765 & 0.2497 & $16.0 \%$ & $0.46[0.28,0.75]$ & & & \\
\hline $\begin{array}{l}\text { Zheng } 2018 \\
\text { Subtotal }(95 \% \mathrm{Cl})\end{array}$ & -0.3425 & 0.3189 & $\begin{array}{l}11.6 \% \\
83.6 \%\end{array}$ & $\begin{array}{l}0.71[0.38,1.33] \\
0.68[0.53,0.88]\end{array}$ & & & \\
\hline \multicolumn{8}{|c|}{$\begin{array}{l}\text { Heterogeneity: } \mathrm{Tau}^{2}=0.02 ; \mathrm{Chi}^{2}=8.00, \mathrm{df}=7(\mathrm{P}=0.33) ; \mathrm{I}^{2}=12 \% \\
\text { Test for overall effect: } \mathrm{Z}=3.01(\mathrm{P}=0.003)\end{array}$} \\
\hline Total $(95 \% \mathrm{Cl})$ & & & $100.0 \%$ & $0.72[0.56,0.93]$ & & & \\
\hline \multicolumn{6}{|c|}{\begin{tabular}{lcc} 
Heterogeneity: $\mathrm{Tau}^{2}=0.04 ; \mathrm{Chi}^{2}=12.25, \mathrm{df}=9(\mathrm{P}=0.20) ; \mathrm{I}^{2}=27 \%$ & \multicolumn{1}{c}{$\begin{array}{l}1 \\
\text { Test for overall effect: } \mathrm{Z}=2.54(\mathrm{P}=0.01)\end{array}$} \\
Test for subqroup differences: $\mathrm{Chi}^{2}=0.59, \mathrm{df}=1(\mathrm{P}=0.44), \mathrm{I}^{2}=0 \%$ & $\quad$ Favours [CCRT+AH]
\end{tabular}} & Favours [CCRT] & 100 \\
\hline
\end{tabular}

Figure 4. Forest plot of disease-free survival for CCRT with AH vs. CCRT alone with subgroup analysis based on study type. CCRT, concurrent chemoradiotherapy; AH, adjuvant hysterectomy; RCT, randomized controlled trial; SE, standard error; IV, inverse variance; df, degrees of freedom.

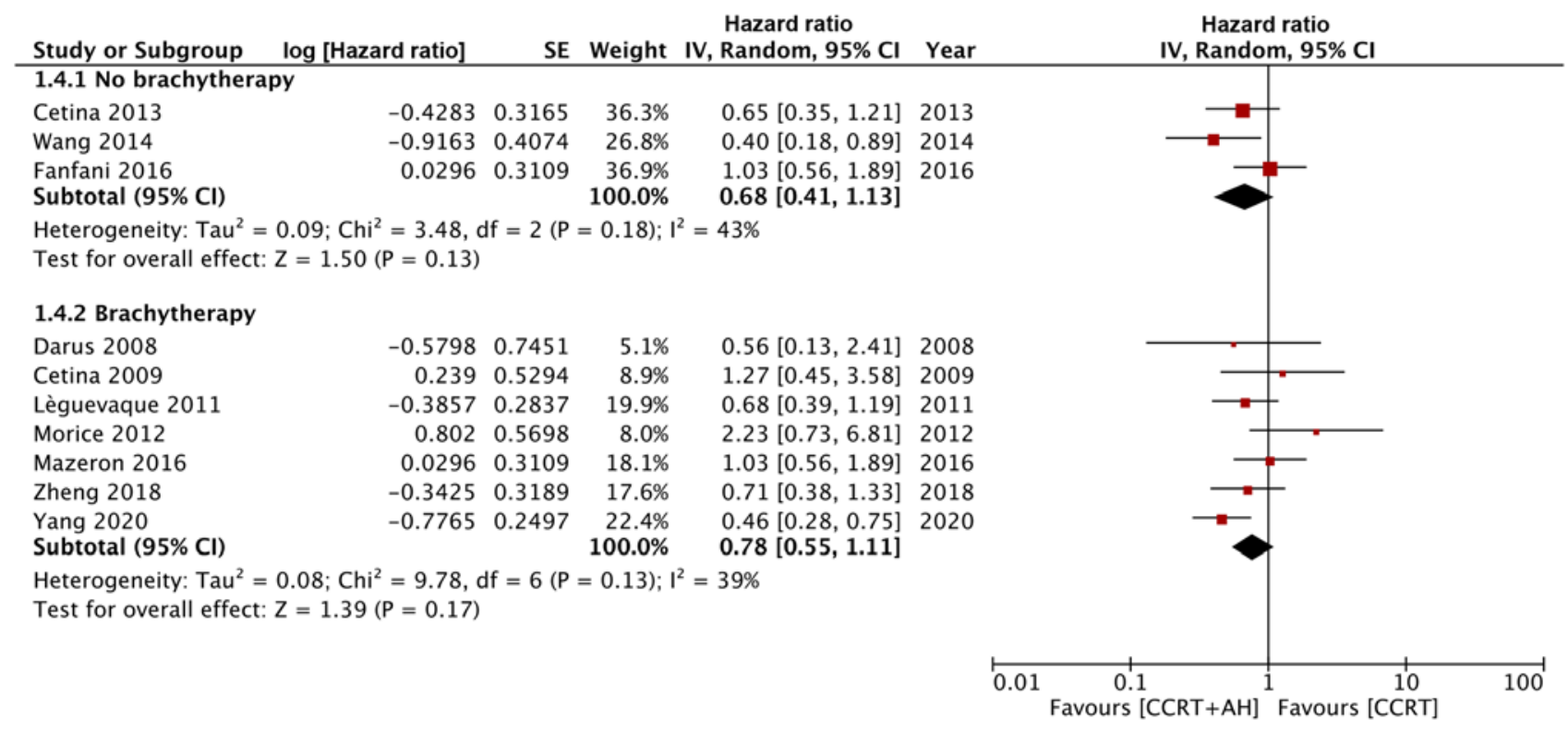

Figure 5. Forest plot of disease-free survival for CCRT with AH vs. CCRT alone with subgroup analysis based on the use of brachytherapy in the CCRT with hysterectomy group. CCRT, concurrent chemoradiotherapy; AH, adjuvant hysterectomy; SE, standard error; IV, inverse variance; df, degrees of freedom.

\section{Discussion}

The utility of additional surgery after CCRT for the management of LACC has remained under considerable debate in the gynecological literature for over two decades $(7,18,22,23)$. While adjuvant hysterectomy has fallen out of favor in the USA (12), a French survey from 2016 reported that one-third of academic centers still perform the procedure in patients with complete response to CCRT and negative paraaortic nodes (26). To date, two RCTs have been performed to compare outcomes for patients treated with or without hysterectomy after CCRT. A trial by Morice et al (32) in France conducted in 2003 included 61 patients but was closed early. The authors reported that since a majority of the treating physicians favored hysterectomies, they were reluctant to enroll patients for the trial. In another study, Cetina et al (31) did not obtain any differences in OS or DFS when comparing patients with patients stage IB2-IIB LACC undergoing external beam radiotherapy (EBRT) with chemotherapy followed by either hysterectomy or brachytherapy. Given the limitations of both of these RCTs, 


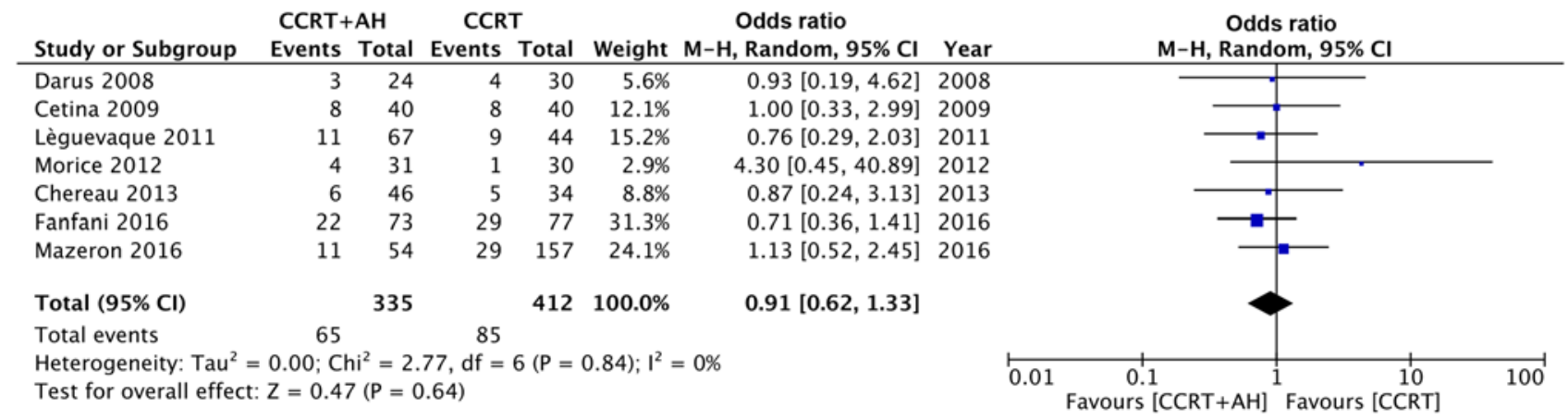

Figure 6. Forest plot of mortality rate for CCRT with AH vs. CCRT alone. CCRT, concurrent chemoradiotherapy; AH, adjuvant hysterectomy; M-H, Mantel-Haentzel; df, degrees of freedom.

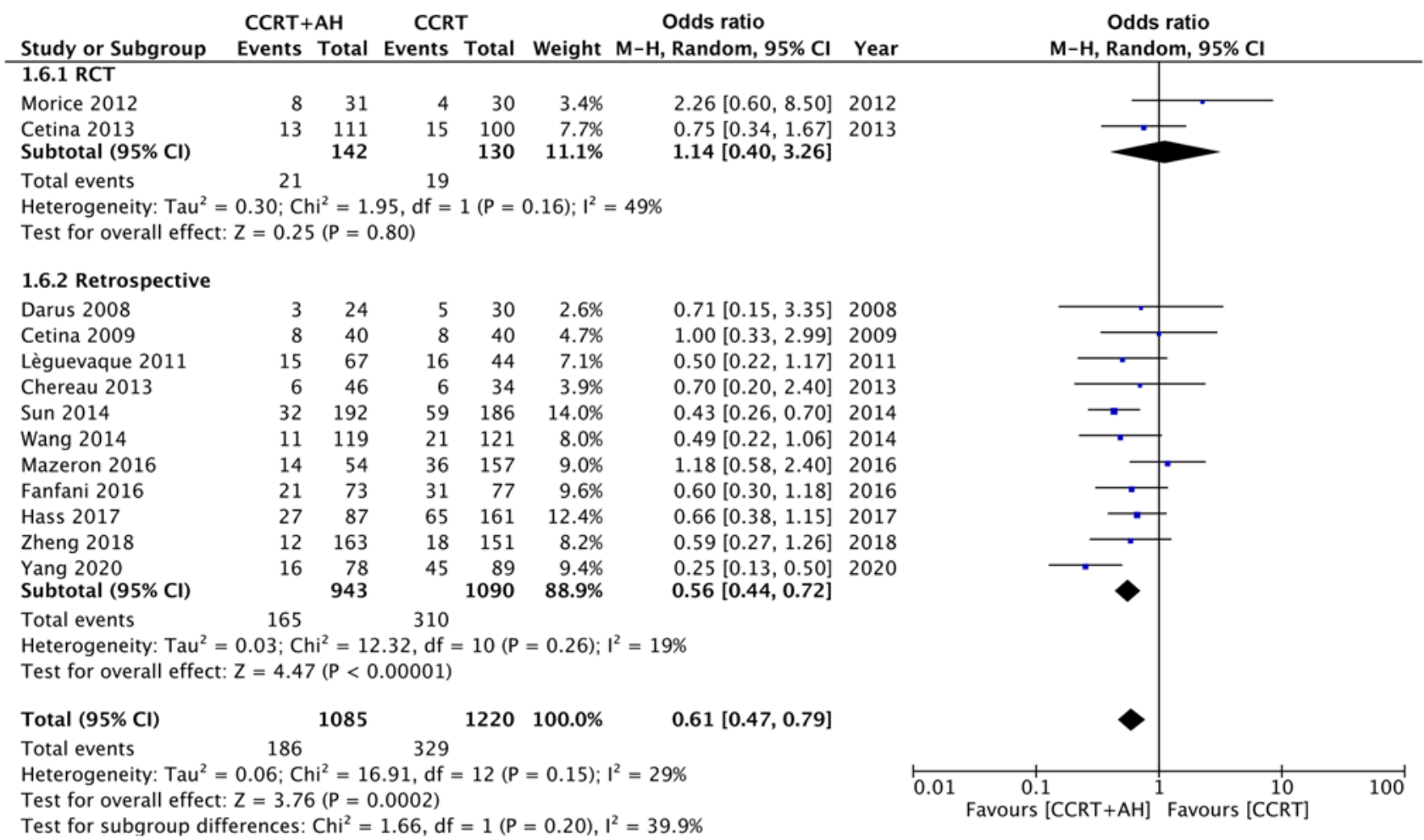

Figure 7. Forest plot of recurrence rate for CCRT with AH vs. CCRT alone with subgroup analysis based on study type. CCRT, concurrent chemoradiotherapy; AH, adjuvant hysterectomy; RCT, randomized controlled trial; M-H, Mantel-Haentzel; df, degrees of freedom.

numerous clinicians have reported their experience regarding the role of adjuvant hysterectomy, but by means of retrospective studies (11,12,24-30,33-35). The present review was performed to provide comprehensive and updated evidence on this subject.

The present analysis of all included studies points to significantly better OS and DFS in patients undergoing hysterectomy after CCRT. By contrast, a previous meta-analysis by Shi et al (10) reported no difference in OS between the two groups (HR, 1.13; 95\% CI, 0.91 to 1.41$)$. This discrepancy may be attributed to two major reasons: First, the study by Shi et al (10) included only pooled data from eight studies and furthermore, in two of those studies, patients were only treated with radiotherapy without concurrent chemotherapy. The results of the present study, however, require to be interpreted with caution considering the instability of the subgroup analyses. The pooled results of RCTs did not demonstrate any significant differences in OS or DFS. However, the results may not be reliable as only 2 RCTs were available for analysis both of small sample size. There was high inter-study heterogeneity and low overall quality. The pooled analysis of retrospective studies did indicate a significantly improved OS and DFS in patients undergoing hysterectomy.

Brachytherapy after EBRT is an important element of CCRT. A combined dose of $\geq 80-85$ Gy of brachytherapy and EBRT is recommended for LACC (36). In the present review, four studies compared hysterectomy as an alternative to brachytherapy after EBRT and chemotherapy. In the absence of brachytherapy, the total radiation dose in patients undergoing CCRT with hysterectomy was lower than that in patients receiving both EBRT and brachytherapy. This leads to baseline differences among these studies that are absent from the comparisons of studies utilizing brachytherapy for 
Table II. Meta-regression for proportion of moderator on the pooled effect size.

\begin{tabular}{|c|c|c|c|c|c|}
\hline \multicolumn{6}{|c|}{ A, Overall survival (hazard ratio) } \\
\hline Moderator & $\beta$ & Lower 95\% CI & Upper 95\% CI & $\mathrm{SE}$ & P-value \\
\hline Stage IB2 & 0.000 & -0.008 & 0.009 & 0.004 & 0.974 \\
\hline Stage II & -0.007 & -0.013 & -0.000 & 0.003 & 0.044 \\
\hline Stage III & 0.005 & -0.000 & 0.011 & 0.003 & 0.069 \\
\hline SCC & 0.007 & -0.001 & 0.015 & 0.004 & 0.069 \\
\hline RH & 0.008 & -0.004 & 0.021 & 0.006 & 0.180 \\
\hline
\end{tabular}

B, Disease free survival (hazard ratio)

\begin{tabular}{lccrr}
\hline Moderator & $\beta$ & Lower 95\% CI & Upper 95\% CI & SE \\
\hline Stage IB2 & 0.008 & -0.005 & 0.020 & 0.007 \\
Stage II & -0.004 & -0.011 & 0.002 & 0.246 \\
Stage III & 0.002 & -0.004 & 0.007 & 0.175 \\
SCC & 0.005 & -0.001 & 0.011 & 0.003 \\
RH & 0.006 & -0.005 & 0.017 & 0.003 \\
& & & 0.006 & 0.121 \\
\hline
\end{tabular}

C, Mortality (odds ratio)

\begin{tabular}{lccrr}
\hline Moderator & $\beta$ & Lower 95\% CI & Upper 95\% CI & PE \\
\hline Stage IB2 & 0.007 & -0.010 & 0.023 & 0.008 \\
Stage II & 0.004 & -0.009 & 0.017 & 0.007 \\
Stage III & -0.004 & -0.013 & 0.005 & 0.024 \\
SCC & -0.004 & -0.030 & 0.023 & 0.004 \\
RH & -0.005 & -0.023 & 0.012 & 0.377 \\
\hline
\end{tabular}

$\mathrm{D}$, Recurrence (odds ratio)

\begin{tabular}{|c|c|c|c|c|c|}
\hline Moderator & $\beta$ & Lower $95 \%$ CI & Upper $95 \%$ CI & SE & P-value \\
\hline Stage IB2 & 0.011 & -0.001 & 0.023 & 0.006 & 0.079 \\
\hline Stage II & -0.004 & -0.011 & 0.003 & 0.004 & 0.270 \\
\hline Stage III & 0.000 & -0.006 & 0.007 & 0.003 & 0.926 \\
\hline SCC & -0.000 & -0.020 & 0.020 & 0.010 & 0.986 \\
\hline $\mathrm{RH}$ & 0.007 & -0.007 & 0.020 & 0.007 & 0.334 \\
\hline
\end{tabular}

SCC, squamous cell carcinoma; RH, radical hysterectomy; CI, confidence interval; SE, standard error. P-values in bold denote significant results. $\beta$ is the meta-regression co-efficient.

both study groups. To eliminate such variation, a subgroup analysis was performed. No significant differences in OS or DFS were identified, irrespective of the use of brachytherapy in the CCRT and hysterectomy group. The insignificant results in these subgroups despite the significant results in the earlier total analysis may be due to the small number of studies pooled. In addition, the excluded data in the study by Albert et al (12) (which did not report data on brachytherapy), with the upper end of the $95 \% \mathrm{CI}$ above 1 (HR, $0.64 ; 95 \% \mathrm{CI}, 0.40$ to 1.01 ) may have contributed to the insignificant results. It may be reasoned that the results of the present meta-analysis with grouping based on the use of brachytherapy provides better evidence than the overall analysis, as studies with similar baseline treatment protocols were pooled in these subgroups.

After pooling data on the absolute number of deaths and recurrences, no significant difference in mortality was observed between the two study groups, but a significantly higher incidence of recurrence was determined in the CCRT only group. Shim et al (8) obtained similar results in their meta-analysis, which also reported no differences in mortality (OR, 1.01; 95\% CI, 0.58 to 1.78 ) but higher rates of recurrence (OR, 0.59; 95\% CI, 0.44 to 0.79) in patients undergoing CCRT without hysterectomy than in those undergoing hysterectomy. On further subgroup analyses, the recurrence rates did not differ for the RCTs, probably due to the small sample size, and they remained significant on subgroup analyses based on the use of brachytherapy. Of note, the present analysis of DFS in the brachytherapy subgroup indicated a lack of difference between the CCRT with hysterectomy and CCRT alone groups. This variation may be due to the addition of the study of Sun et al (30), which reported a significant difference in recurrence rates [CCRT with hysterectomy (16.6\%) vs. CCRT alone (31.7\%)] between the two groups, in the latter analysis.

One of the biggest limitations when assessing the benefit of adjuvant hysterectomy regarding clinical outcomes after CCRT is the influence of confounding factors. The bias is further amplified in the present results, as 12 of the 14 studies included in the analysis were retrospective in nature. Several 


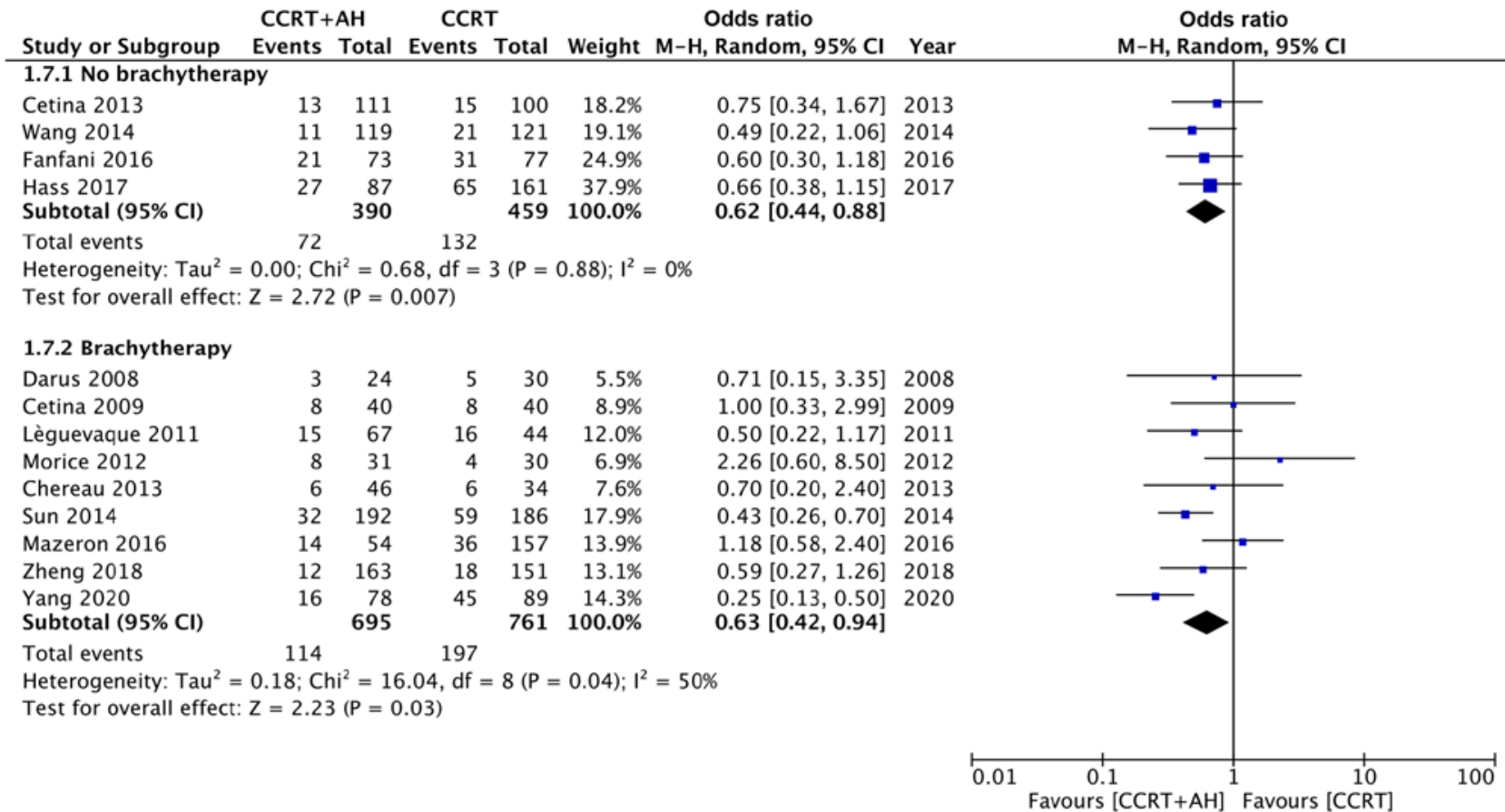

Figure 8. Forest plot of recurrence rate for CCRT with AH vs. CCRT alone with subgroup analysis based on the use of brachytherapy in the CCRT with hysterectomy group. CCRT, concurrent chemoradiotherapy; AH, adjuvant hysterectomy; M-H, Mantel-Haentzel; df, degrees of freedom.

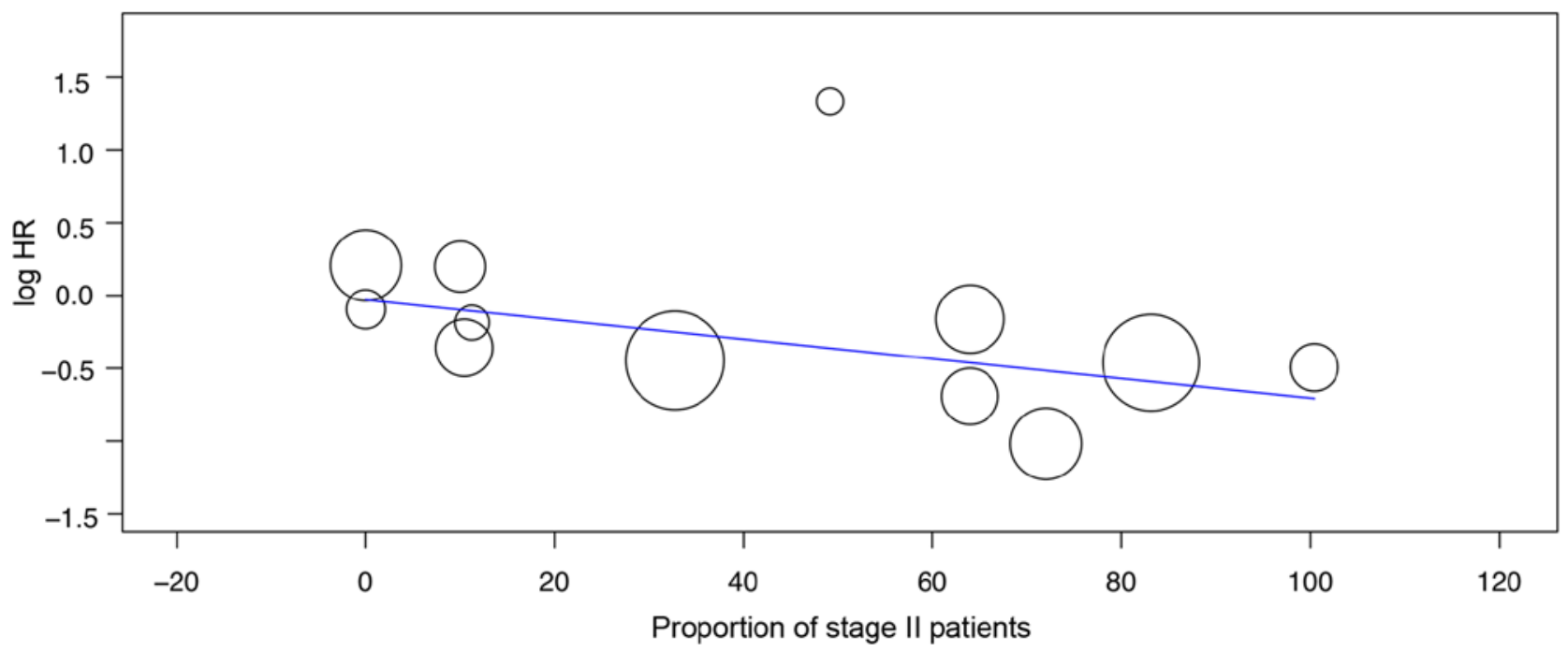

Figure 9. Meta-regression plot for the influence of the proportion of patients with stage II locally-advanced cervical cancer on the logarithmic HR of overall survival. Individual studies are denoted by circles. The diameter of the circle denotes the weight of the study. HR, hazard ratio.

factors, such as the presence of comorbidities, tumor size, tumor stage, histology, residual disease after CCRT and lymph node involvement may influence the decision to perform completion hysterectomy $(11,12,24,27)$. Albert et al (12) demonstrated that patients with stage IIA2 disease were less likely to undergo hysterectomy, whereas patients with adenocarcinoma (which is not as radiosensitive as SCC and has worse prognosis than SCC) were more likely to undergo adjuvant surgery. Furthermore, the use of radical vs. simple hysterectomy may also influence survival and recurrence rates (37). In the meta-regression analysis, a significant association between the proportion of stage II disease and OS was determined. The OS increased as the proportion of patients with stage II disease increased. This may be attributed to the fact that adjuvant hysterectomy after CCRT failure is easier to perform in patients with stage II disease and may thereby account for the better survival (10-12). In addition, it was not possible to subdivide the present data according to the disease stage due to the lack of adequate data from the included studies. No other influence by the LACC stage, tumor histology or history of radical hysterectomy on the pooled effect size was observed.

The presence of residual disease after CCRT is a major concern and has been linked to local disease progression and poor OS $(38,39)$. According to certain clinicians, adjuvant hysterectomy may remove residual tumor masses, thereby improving local control $(11,25)$. Identification of patients with 
residual disease after CCRT is generally based on clinical examination, MRI or cervical biopsy results $(40,41)$. However, these methods are less accurate than surgical-pathology confirmation. Yang et al (11) reported residual tumors in $27.4 \%$ of patients after CCRT based on imaging studies in their cohort, but $64.1 \%$ of patients had residual tumor on the final post-hysterectomy pathological examination. In the present study, no meta-regression analysis was performed to assess the influence of post-CCRT residual disease on pooled outcomes, as only six of the included studies had reported these data.

Of note, the present meta-analysis had certain limitations. First, as discussed earlier, the results are mostly based on data from unmatched retrospective studies. The possibility of selection bias having influenced the present results cannot be ruled out. The HRs were only adjusted for confounding variables using multivariate regression analysis in two of the studies included $(11,12)$. In addition, only two RCTs were included $(31,32)$, which was insufficient to determine any significant differences. Furthermore, in most studies, HR values were extrapolated from Kaplan-Meier survival curves and not from the original data. The calculation of data from survival curves is prone to errors (16). In addition, variations amongst the included studies in patient populations, and variables including CCRT doses, surgical techniques and follow-up duration may have skewed the present. Therefore, a random-effects model was used to account for such inter-study variations. As another limitation, meta-regression was not performed for all confounding factors, such as disease sub-stages, lymph node metastasis or residual disease after CCRT due to lack of data. Finally, the quality of the included studies was not high, which limited our ability to draw strong conclusions.

However, compared to previous studies $(8,10)$, the present review provides a significant update with the addition of six new studies. Both HR and OR data were pooled with appropriate subgroup and meta-regression analyses to provide a comprehensive review. The stability of the results according to the sensitivity analysis lends credibility to the present results.

To conclude, despite the overall analysis indicating improved OS and DFS with the use of adjuvant hysterectomy after CCRT, subgroup analyses of studies based on similar treatment protocols failed to demonstrate any significant benefit of hysterectomy post-CCRT for patients with LACC. However, it was indicated that the recurrence rate may be higher in patients undergoing CCRT without hysterectomy. In addition, analysis of retrospective studies indicated significantly improved OS and DFS in patients undergoing hysterectomy compared with patients not undergoing the adjuvant surgery. The limited quality of studies and selection bias from retrospective data restrict our ability to draw conclusions. Further high-quality, homogenous RCTs are required to derive stronger evidence. In addition, future studies should provide detailed analyses regarding the use of adjuvant hysterectomy based on different tumor histologies, tumor stages, amounts of residual disease and different doses of total radiotherapy to confirm or disprove the benefit of hysterectomy in these patients.

\section{Acknowledgements}

Not applicable.

\section{Funding}

No funding was received.

\section{Availability of data and materials}

The datasets used and/or analyzed during the current study are available from the corresponding author on reasonable request.

\section{Authors' contributions}

WL conceived and designed the study. CL, ZY and LG collected the data and performed the literature search. WL was involved in the writing of the manuscript. All authors read and approved the final manuscript.

\section{Ethics approval and consent to participate}

Not applicable.

\section{Patient consent for publication}

Not applicable.

\section{Competing interests}

The authors declare that they have no competing interests.

\section{References}

1. Bray F, Ferlay J, Soerjomataram I, Siegel RL, Torre LA and Jemal A: Global cancer statistics 2018: GLOBOCAN estimates of incidence and mortality worldwide for 36 cancers in 185 countries. CA Cancer J Clin 68: 394-424, 2018.

2. Shrestha AD, Neupane D, Vedsted P and Kallestrup P: Cervical cancer prevalence, incidence and mortality in low and middle income countries: A systematic review. Asian Pac J Cancer Prev 19: 319-324, 2018.

3. Pecorelli S, Zigliani L and Odicino F: Revised FIGO staging for carcinoma of the cervix. Int J Gynecol Obstet 105: 107-108, 2009.

4. Haie-Meder C, Morice P and Castiglione M; ESMO Guidelines Working Group: Cervical cancer: ESMO clinical recommendations for diagnosis, treatment and follow-up. Ann Oncol (20 Suppl 4): S27-S28, 2009.

5. Tanderup K, Lindegaard JC, Kirisits C, Haie-Meder C, Kirchheiner K, de Leeuw A, Jürgenliemk-Schulz I, Van Limbergen E and Pötter R: Image Guided Adaptive Brachytherapy in cervix cancer: A new paradigm changing clinical practice and outcome. Radiother Oncol 120: 365-369, 2016.

6. Fanfani F, Fagotti A, Ferrandina G, Raspagliesi F, Ditto A, Cerrotta AM, Morganti A, Smaniotto D and Scambia G: Neoadjuvant chemoradiation followed by radical hysterectomy in FIGO Stage IIIB cervical cancer: Feasibility, complications, and clinical outcome. Int J Gynecol Cancer 19: 1119-1124, 2009.

7. Platt SL, Patel A, Humphrey PJ, Al-Booz H and Bailey J: Completion surgery after chemoradiotherapy for cervical cancer -is there a role? UK Cancer Centre experience of hysterectomy post chemo-radiotherapy treatment for cervical cancer. J Obstet Gynaecol 39: 68-73, 2019.

8. Shim SH, Kim SN, Chae SH, Kim JE and Lee SJ: Impact of adjuvant hysterectomy on prognosis in patients with locally advanced cervical cancer treated with concurrent chemoradiotherapy: A meta-analysis. J Gynecol Oncol 29: e25, 2018.

9. Yang J, Shen K, Wang J, Yang J and Cao D: Extrafascial hysterectomy after concurrent chemoradiotherapy in locally advanced cervical adenocarcinoma. J Gynecol Oncol 27: e40, 2016.

10. Shi D, Liang Z, Zhang C, Zhang $\mathrm{H}$ and Liu X: The effect of surgery on the survival status of patients with locally advanced cervical cancer after radiotherapy/chemoradiotherapy: A meta-analysis. BMC Cancer 18: 308, 2018. 
11. Yang J, Yang J, Cao D, Shen K, Ma J and Zhang F: Completion hysterectomy after chemoradiotherapy for locally advanced adeno-type cervical carcinoma: Updated survival outcomes and experience in post radiation surgery. J Gynecol Oncol 31: e16, 2020.

12. Albert A, Allbright R, Lee A and Vijayakumar S: Preoperative chemoradiation followed by hysterectomy for cervical cancer: Patterns of care and survival in a large, hospital database. J Gynecol Oncol 30: e41, 2019.

13. Moher D, Liberati A, Tetzlaff $\mathbf{J}$ and Altman DG; PRISMA Group: Preferred reporting items for systematic reviews and meta-analyses: The PRISMA statement. BMJ 339: b2535, 2009.

14. Higgins JPT, Altman DG and Sterne JAC (editors): Chapter 8: Assessing risk of bias in included studies. In: Higgins JPT, Churchill R, Chandler J, Cumpston MS (eds), Cochrane Handbook for Systematic Reviews of Interventions version 5.2.0 (updated June 2017), Cochrane, 2017. Available from www. training.cochrane.org/handbook.

15. Kim SY, Park JE, Lee YJ, Seo HJ, Sheen SS, Hahn S, Jang BH and Son HJ: Testing a tool for assessing the risk of bias for nonrandomized studies showed moderate reliability and promising validity. J Clin Epidemiol 66: 408-414, 2013.

16. Tierney JF, Stewart LA, Ghersi D, Burdett S and Sydes MR: Practical methods for incorporating summary time-to-event data into meta-analysis. Trials 8: 16, 2007.

17. Wallace BC, Schmid CH, Lau J and Trikalinos TA: Meta-Analyst: Software for meta-analysis of binary, continuous and diagnostic data. BMC Med Res Methodol 9: 80, 2009.

18. Li H, Pang Y and Cheng X: Surgery of primary sites for stage IVB cervical cancer patients receiving chemoradiotherapy: A population-based study. J Gynecol Oncol 31: e8, 2020.

19. Gosset M, Chargari C, Bentivegna E, Leary A, Genestie C, Maulard A, Morice P and Gouy S: Should we cease to perform salvage hysterectomy after chemoradiation and brachytherapy in locally advanced cervical cancer? Anticancer Res 39: 2919-2926, 2019.

20. Haque W, Verma V, Butler EB and Teh BS: Utilization of hysterectomy following chemoradiation for IB2/IIA2 cervical cancer in the national cancer data base. Anticancer Res 38: 3175-3179, 2018.

21. Huang XB, Zhang WW, Wu SG, Sun JY, He ZY and Zhou J: Survival benefits with the addition of adjuvant hysterectomy to radiochemotherapy for treatment of stage I-II adenocarcinoma of the uterine cervix. J Surg Oncol 118: 574-580, 2018.

22. Keys HM, Bundy BN, Stehman FB, Okagaki T, Gallup DG, Burnett AF, Rotman MZ and Fowler WC Jr: Radiation therapy with and without extrafascial hysterectomy for bulky stage IB cervical carcinoma: A randomized trial of the Gynecologic Oncology Group. Gynecol Oncol 89: 343-353, 2003.

23. Perez CA, Grigsby PW, Camel HM, Galakatos AE, Mutch D and Lockett MA: Irradiation alone or combined with surgery in stage IB, IIA and IIB carcinoma of uterine cervix: Update of a nonrandomized comparison. Int J Radiat Oncol Biol Phys 31: 703-716, 1995.

24. Zheng D, Mou HP, Diao P, Li XM, Zhang CL, Jiang J, Chen JL, Wang LS, Wang Q, Zhou GY, et al: Chemoradiotherapy in combination with radical surgery is associated with better outcome in cervical cancer patients. Oncotarget 9: 2866-2875, 2017.

25. Hass P, Eggemann H, Costa SD and Ignatov A: Adjuvant hysterectomy after radiochemotherapy for locally advanced cervical cancer. Strahlenther Onkol 193: 1048-1055, 2017.

26. Mazeron R, Gouy S, Chargari C, Rivin Del Campo E, Dumas I, Mervoyer A, Genestie C, Bentivegna E, Balleyguier C, Pautier P, et al: Post radiation hysterectomy in locally advanced cervical cancer: Outcomes and dosimetric impact. Radiother Oncol 120: 460-466, 2016

27. Fanfani F, Vizza E, Landoni F, de Iaco P, Ferrandina G, Corrado G, Gallotta V, Gambacorta MA, Fagotti A, Monterossi G, et al: Radical hysterectomy after chemoradiation in FIGO stage III cervical cancer patients versus chemoradiation and brachytherapy: Complications and 3-years survival. Eur J Surg Oncol 42: 1519-1525, 2016

28. Chereau E, DE LA Hosseraye C, Ballester M, Monnier L, Rouzier R, Touboul E and Daraï E: The role of completion surgery after concurrent radiochemotherapy in locally advanced stages IB2-IIB cervical cancer. Anticancer Res 33: 1661-1666, 2013.
29. Wang N, Li WW, Li JP, Liu JY, Zhou YC, Zhang Y, Hu J, Huang YH, Chen Y, Wei LC, et al: Comparison of concurrent chemoradiotherapy followed by radical surgery and high-dose-rate intracavitary brachytherapy: A retrospective study of 240 patients with FIGO stage IIB cervical carcinoma. Onco Targets Ther 7: 91-100, 2014

30. Sun L, Sheng X, Jiang J, Li X, Liu N, Liu Y, Zhang T, Li D, Zhang $X$ and Wei P: Surgical morbidity and oncologic results after concurrent chemoradiation therapy for advanced cervical cancer. Int J Gynaecol Obstet 125: 111-115, 2014

31. Cetina L, González-Enciso A, Cantú D, Coronel J, Pérez-Montiel D, Hinojosa J, Serrano A, Rivera L, Poitevin A, Mota A, et al: Brachytherapy versus radical hysterectomy after external beam chemoradiation with gemcitabine plus cisplatin: A randomized, phase III study in IB2-IIB cervical cancer patients. Ann Oncol 24: 2043-2047, 2013.

32. Morice P, Rouanet P, Rey A, Romestaing P, Houvenaeghel G, Boulanger JC, Leveque J, Cowen D, Mathevet P, Malhaire JP, et al: Results of the GYNECO 02 study, an FNCLCC phase III trial comparing hysterectomy with no hysterectomy in patients with a (clinical and radiological) complete response after chemoradiation therapy for stage IB2 or II cervical cancer. Oncologist 17: 64-71, 2012.

33. Lèguevaque P, Motton S, Delannes M, Querleu D, Soulé-Tholy M, Tap G and Houvenaeghel G: Completion surgery or not after concurrent chemoradiotherapy for locally advanced cervical cancer? Eur J Obstet Gynecol Reprod Biol 155: 188-192, 2011.

34. Cetina L, Garcia-Arias A, Candelaria M, Cantú D, Rivera L, Coronel J, Bazan-Perkins B, Flores V, Gonzalez A and Dueñas-González A: Brachytherapy versus radical hysterectomy after external beam chemoradiation: A non-randomized matched comparison in IB2-IIB cervical cancer patients. World J Surg Oncol 7: 19, 2009.

35. Darus CJ, Callahan MB, Nguyen QN, Pastore LM, Schneider BF, Rice LW and Jazaeri AA: Chemoradiation with and without adjuvant extrafascial hysterectomy for IB2 cervical carcinoma. Int J Gynecol Cancer 18: 730-735, 2008.

36. Gaffney DK, Erickson-Wittmann BA, Jhingran A, Mayr NA, Puthawala AA, Moore D, Rao GG, Small W Jr, Varia MA, Wolfson AH, et al: ACR Appropriateness Criteria ${ }^{\circledR}$ on advanced cervical cancer expert panel on radiation oncology-gynecology. Int J Radiat Oncol Biol Phys 81: 609-614, 2011.

37. Boers A, Arts HJG, Klip H, Nijhuis ER, Pras E, Hollema H, Wisman GB, Nijman HW, Mourits MJ, Reyners AK, et al: Radical surgery in patients with residual disease after (chemo) radiation for cervical cancer. Int $\mathrm{J}$ Gynecol Cancer 24: 1276-1285, 2014.

38. Houvenaeghel G, Lelievre L, Buttarelli M, Jacquemier J, Carcopino X, Viens P and Gonzague-Casabianca L: Contribution of surgery in patients with bulky residual disease after chemoradiation for advanced cervical carcinoma. Eur J Surg Oncol 33: 498-503, 2007.

39. Azria E, Morice P, Haie-Meder C, Thoury A, Pautier P, Lhomme C, Duvillard P and Castaigne D: Results of hysterectomy in patients with bulky residual disease at the end of chemoradiotherapy for stage IB2/II cervical carcinoma. Ann Surg Oncol 12: 332-337, 2005.

40. Nijhuis ER, van der Zee AGJ, in 't Hout BA, Boomgaard JJ, de Hullu JA, Pras E, Hollema H, Aalders JG, Nijman HW, Willemse PH and Mourits MJ: Gynecologic examination and cervical biopsies after (chemo) radiation for cervical cancer to identify patients eligible for salvage surgery. Int J Radiat Oncol Biol Phys 66: 699-705, 2006.

41. Gui B, Valentini AL, Miccò M, D'Agostino GR, Tagliaferri L, Zannoni GF, Fanfani F, Manfredi R and Bonomo L: Cervical cancer response to neoadjuvant chemoradiotherapy: MRI assessment compared with surgery. Acta radiol 57: 1123-1131, 2016.

This work is licensed under a Creative Commons Attribution-NonCommercial-NoDerivatives 4.0 International (CC BY-NC-ND 4.0) License. 